\title{
Evaluación del nuevo enfoque en atención a la cronicidad en las organizaciones sanitarias integradas en el País Vasco
}

\author{
Evaluation of the new integrated care approach \\ in the Basque Country
}

\author{
N. Toro Polancoํㅜ P. Vázquez Pérez ${ }^{2}$, R. Nuño Solinís ${ }^{1}$, J.J. Mira Solves ${ }^{3}$
}

\section{RESUMEN}

Fundamento. En el año 2010 Euskadi puso en marcha la "Estrategia para afrontar el reto de la cronicidad" tratando de reorientar las organizaciones sanitarias hacia modelos más integrados que proporcionaran una respuesta de calidad y más eficiente al paciente crónico. Se han creado cuatro Organizaciones Sanitarias Integradas (OSI) que suponen la integración en una única organización de los dos niveles asistenciales: atención primaria y especializada. El objetivo del estudio es conocer el grado de desarrollo alcanzado por las cuatro OSI en su estrategia de gestión integrada de la cronicidad tomando como referente de la evaluación el Chronic Care Model.

Material y métodos. Autoevaluación en las cuatros OSI con la herramienta IEMAC por parte del equipo directivo de cada organización.

Resultados. La puntuación promedio es de 16 puntos sobre 100 , lo que implica que las organizaciones están emprendiendo planes de acción en el ámbito de la gestión de la atención integrada, pero con un despliegue limitado y sin que se puedan aportar evidencias de evaluación de los resultados de las acciones puestas en marcha. La dimensión mejor valorada fue "Organización del Sistema de Salud", mientras que la menos valorada fue "Salud Comunitaria".

Conclusiones. Las OSI son motores del cambio para lograr una atención integral a la cronicidad. Los aspectos relacionados con la integración de recursos comunitarios y la evaluación de resultados constituyen dos de las áreas de mejora para desplegar el conjunto de intervenciones propuestas en IEMAC. Los cambios organizativos que supone el CCM requieren de períodos de tiempo superiores a dos años.

Palabras clave. Evaluación de servicios sanitarios. Sistema sanitario integrado. Cronicidad. Calidad.

\begin{abstract}
Background. In 2010 the Basque Government launched its "Strategy for tackling the challenge of chronicity in the Basque Country", in order to transform healthcare organisations into more integrated care models, with the aim of improving quality and efficiency in chronicity management. Four Integrated Healthcare Organisations (IHOs) were created to unify primary and specialised care into one single organisation. The aim of this stu$\mathrm{dy}$ is to measure the degree of readiness of these IHOs to cope with chronicity, using the Chronic Care Model (CCM) as a reference.
\end{abstract}

Material and methods. Self-assessment processes using ARCHO (Assessment of Readiness for Chronicity in Health Care Organisations) were carried out in four IHOs by the management teams.

Results. The average score was 16 out of 100 points, which signals that healthcare organisations are undertaking action plans in the realm of integrated care, but with limited deployment and without a systematic process for evaluating outcomes. The dimension that ranks best is "Organization of the health system", while "Community health" has the lowest ranking.

Conclusions. IHOs are the key for achieving integrated care for chronic illnesses. Integration of community resources and evaluation of results are two of the fields that need to be improved in order to achieve the set of interventions proposed in ARCHO. The organisational changes involved in the CCM require periods longer than two years.

Keywords. Health services assessment. Integrated health system. Chronicity. Quality.
1. Instituto Vasco de Innovación Sanitaria (O+berri).

2. Socióloga. Investigadora en ciencias sociales. Instituto Vasco de Innovación Sanitaria (O+berri).

3. Departament de Salut Sant Joan-Alacant, Consellería de Sanitat. Universitat Miguel Hernández. Elche. Alicante. España.

\section{Correspondencia:}

Nuria Toro Polanco

Instituto Vasco de Innovación Sanitaria (O+berri)

Plaza Asua, 1

48150 Sondika (Bizkaia)

E-mail: toro@bioef.org 


\section{INTRODUCCIÓN}

El fenómeno de la cronicidad, entendido como un marco amplio de comprensión de las condiciones crónicas sobre quien las padece, su entorno, la comunidad, el sistema sanitario y la sociedad, induce a repensar el modelo de prestación de servicios sanitarios y sociales y el rol que pueden desempeñar sus diferentes agentes ${ }^{1}$. La creciente presencia de pluripatología y su mayor complejidad como fenómenos asociados a la cronicidad suponen retos adicionales para las organizaciones sanitarias y para los sistemas de generación de conocimiento clínico ${ }^{2,3}$.

Por todo ello, existe un amplio consenso y una senda de avance hacia nuevos modelos asistenciales ${ }^{4}$, diferentes a los actuales, buscando una atención más proactiva, continuada e integrada, centrada en el paciente. La reciente difusión de la Estrategia en Cronicidad del Sistema Nacional de Salud $^{5}$ se suma al rediseño de los modelos asistenciales que, en los últimos años, se vienen observando.

La sanidad pública vasca no ha sido ajena a esta necesidad y el Departamento de Sanidad y Consumo del Gobierno Vasco puso en marcha en 2010 la "Estrategia para afrontar el reto de la cronicidad en Euskadi" ${ }^{\prime \prime}$. Se trata de una apuesta por reorientar las organizaciones sanitarias hacia modelos más integrados para proporcionar una respuesta más eficiente a las necesidades del paciente crónico ${ }^{7}$. Así por ejemplo, la política denominada "Continuo de Atención" busca "garantizar el continuo asistencial mediante el impulso de una asistencia multidisciplinar, coordinada e integrada entre los diferentes servicios, niveles de atención y sectores" a partir de fórmulas que fomenten la colaboración e integración entre atención primaria (AP) y asistencia especializada (AE).

En enero de 2011, se constituyó la Organización Sanitaria Integrada Bidasoa (OSI Bidasoa), que supuso la integración en una única organización de los dos niveles asistenciales, $\mathrm{AP}$ y $\mathrm{AE}$, como alternativa a la pérdida de continuidad asistencial y a los problemas de ineficiencia que suponen los modelos fragmentados existentes en los sistemas sanitarios tradicionales ${ }^{8}$. Meses más tarde, en octubre del 2011, se crearon tres Organizaciones Sanitarias Integradas más ${ }^{9}$ (OSI Alto Deba, OSI Bajo Deba y OSI Goierri-Alto Urola) en pos de la integración y coordinación de la atención que reciben los pacientes desde distintas fuentes de provisión, convirtiendo al paciente en el núcleo central para la configuración de los servicios sanitarios.

En la práctica, las OSI suponen la creación de una estructura común entre un hospital comarcal y los centros de AP de un área determinada, bajo una gobernanza única y objetivos y sistemas de gestión comunes. La coordinación entre niveles asistenciales se consigue a través de iniciativas de integración clínica consensuadas por las dos estructuras integradas que habitualmente toman la forma de rutas asistenciales en las que las tareas y roles en torno al paciente quedan explícitamente recogidos. La gestión económica también es común, favoreciendo la búsqueda de eficiencia a través de la integración de cuidados y la eliminación de duplicidades ${ }^{10}$. Todas estas características posicionan adecuadamente a las OSI hacia la gestión integrada de la cronicidad.

A pesar de que los marcos conceptuales de atención integrada y de atención a la cronicidad vienen convergiendo ${ }^{11}$, la evaluación de cómo las organizaciones se orientan al paciente crónico no es habitual en nuestro medio.

El modelo más reconocido en la gestión de crónicos es el denominado Chronic Care Model $^{12}$ (CCM), siendo además el más respaldado por la evidencia científica ${ }^{13} \mathrm{e}$ inspirador de otros modelos, como el Innovative Care for Chronic Conditions ${ }^{14}$ y el Expanded $\mathrm{CCM}^{15}$. Del CCM se derivan dos instrumentos, ACIC $^{16}$ y Matriz Rand ${ }^{17}$, desarrollados en Estados Unidos. En Europa, también se han desarrollado herramientas de medición a partir del CCM y sus derivados, tales como el Questionnaire of Chronic Illness Care in Primary Care $^{18}$ (QCPC) y el ACIC-G ${ }^{19}$ en Alemania, mientras que en Holanda se ha desarrollado una versión reducida del $\mathrm{ACIC}^{20} \mathrm{y}$ una herramienta propia denomina- 
da Development Model for Integrated Care $^{21}$. Todos estos instrumentos permiten medir el grado de implementación de prácticas que fomentan la gestión integrada de la cronicidad. En España, inspirado en el modelo CCM y en otros instrumentos como el ACIC y la Matriz Rand, se ha desarrollado y validado desde y para un contexto de Sistema Nacional de Salud, IEMAC ${ }^{22}$ (Instrumento de Evaluación de Modelos de Atención ante la Cronicidad).

Estos instrumentos facilitan la puesta en práctica de enfoques efectivos de atención a la cronicidad y la evaluación de sus resultados. Comparten la autoevaluación como enfoque metodológico y la idea de que para poder avanzar hacia la provisión excelente de atención de la cronicidad se requiere conocer el estado de las distintas organizaciones sanitarias, sabiendo qué se hace y cómo se hace.

El objetivo de este estudio es conocer el grado de desarrollo alcanzado por las cuatro OSI existentes en el País Vasco en su estrategia de gestión integrada de la cronicidad tomando como referente de la evaluación el Chronic Care Model (CCM).

\section{MATERIAL Y MÉTODOS}

Se ha llevado a cabo un proceso de autoevaluación en las cuatro organizaciones sanitarias integradas del País Vasco con la herramienta IEMAC.

IEMAC $^{23}$ es un instrumento de autoevaluación que ayuda a las organizaciones a identificar en qué punto se encuentran respecto a la implantación de modelos orientados a la gestión de la atención integrada al paciente crónico, facilitando la identificación de los puntos fuertes y de los precisan una mejora, permitiendo establecer a clínicos y gestores sanitarios una hoja de ruta en la gestión de la cronicidad. IEMAC consta de 6 dimensiones (al igual que el CCM): organización del sistema de salud, salud comunitaria, modelo asistencial, autocuidado, apoyo en la toma de decisiones clínicas y sistemas de información. Estas dimensiones son los ejes de transformación necesarios que propician la adopción de un modelo de atención a la cronicidad.
De estas dimensiones, se derivan 27 componentes y 80 intervenciones. Cada una de estas intervenciones tiene la misma importancia relativa en el instrumento, por lo que no están ponderadas. Su implantación es más efectiva cuando las intervenciones se llevan a cabo de manera combinada, y no se implementan de forma aislada.

La implantación de las intervenciones que componen el instrumento se evalúa en una escala que combina despliegue, evaluación sistemática y orientación a la mejora, en un rango de 0 a 100 , dividida en 5 segmentos:

- Segmento 1 (0-10): Plan de acción o actuaciones aisladas. Despliegue muy limitado (corresponde a estudios pilotos o diseño de proyectos).

- Segmento 2 (15-35): Plan de acción en marcha, sin evaluación. Despliegue de un 25\% (supone que las intervenciones puestas en marcha solo están presentes en algunos centros, cuentan con algunos profesionales y se centran en algunas patologías).

- Segmento 3 (40-60): Plan desarrollándose de forma sistemática. Se evalúan resultados. Despliegue de un $50 \%$ (corresponde a planes e intervenciones ya puestos en práctica en la mayoría de centros, con un amplio número de profesionales, que abarcan a distintas patologías y de los que se cuentan con los primeros resultados de evaluación).

- Segmento 4 (65-85): Plan de acción evaluado de forma sistemática al menos durante dos años. Despliegue de un $75 \%$ (La medición de resultados se viene haciendo de forma sistemática).

- Segmento 5 (90-100): Plan de acción integrado en el modelo asistencial. Despliegue superior al $85 \%$. Se favorece la innovación (corresponde a la revisión de planes de actuación merced a la revisión sistemática de resultados, lo que provoca mejoras sustanciales y oportunidades para desarrollar nuevas intervenciones merced a la capacidad de innovación que favorece compartir el conocimiento clínico adquirido). 
La puntuación total de IEMAC corresponde a la suma de las puntuaciones en cada una de las intervenciones. La puntuación facilita realizar comparaciones a lo largo del tiempo de la propia organización. De forma operativa, puede obtenerse información del grado de desarrollo en cada componente y dimensión, a partir de la media de sus puntuaciones respectivas.

Los procesos de autoevaluación se han llevado a cabo por miembros del equipo directivo, participando en todos los casos el director gerente, el director médico y el director de enfermería de cada OSI. En dos organizaciones se ha contado además con la participación del director de personal, del responsable de calidad, y de profesionales de la Unidad de Gestión Sanitaria. La selección de los participantes se basó en su mayor conocimiento global de los enfoques y estrategias de trabajo en la comarca, de las diferentes intervenciones llevadas a cabo y de sus resultados, ajustando este perfil a las recomendaciones para conducir la autoevaluación del propio IEMAC ${ }^{23}$.

La autoevaluación de la OSI Alto Deba abarcó las actuaciones realizadas en 2011 y las OSI Goierri Alto Urola, Bidasoa y Debabarrena evaluaron las actuaciones implantadas en los años 2011 y 2012.

Cada autoevaluación ha tenido lugar en una sesión de trabajo grupal de una duración aproximada de 4,5 horas. Las reunio- nes se celebraron en la propia OSI objeto de evaluación. La dinámica de la sesión consistió en la presentación del instrumento por parte de un moderador de O+berri, quien explicó el significado de cada una de las 80 intervenciones de IEMAC. Para cada intervención, cada miembro del equipo propuso de forma individual una puntuación en escala 0 a 100, argumentada en base a iniciativas, proyectos y experiencias que se hubieran llevado a cabo en sus organizaciones. La puntuación final para cada intervención fue fruto del consenso de todos los participantes. Toda la información fue codificada por el moderador de las sesiones de trabajo.

Para cada OSI, se han recogido las puntuaciones en las 80 intervenciones de IEMAC, junto con la relación de planes y proyectos llevados a cabo por cada OSI que las justificaban. A partir de las puntuaciones obtenidas en las 80 intervenciones, se han extraído las medias por componente y dimensión para cada OSI. Para el cálculo de las puntuaciones promedio se han seguido las indicaciones del propio instrumento de evaluación ${ }^{23}$.

\section{RESULTADOS}

Las puntuaciones conjunto de las OSI en cada una de las 6 dimensiones de IEMAC se muestran en la tabla 1. Las puntuaciones de cada OSI en la figura 1.

Tabla 1. Puntuaciones promedio en las seis dimensiones de IEMAC para el conjunto de las OSI.

\begin{tabular}{lc}
\hline \multicolumn{1}{c}{ Dimensión } & Puntuación promedio \\
\hline 1. Organización del sistema de salud & 23 \\
\hline 2. Salud comunitaria & 7 \\
\hline 3. Modelo asistencial & 20 \\
\hline 4. Autocuidado & 14 \\
\hline 5. Apoyo en la toma de decisiones clínicas & 15 \\
\hline 6. Sistemas de información & 17 \\
\hline Promedio dimensiones & $\mathbf{1 6}$ \\
\hline
\end{tabular}

Rango de puntuaciones en cada dimensión 0 a 100. 


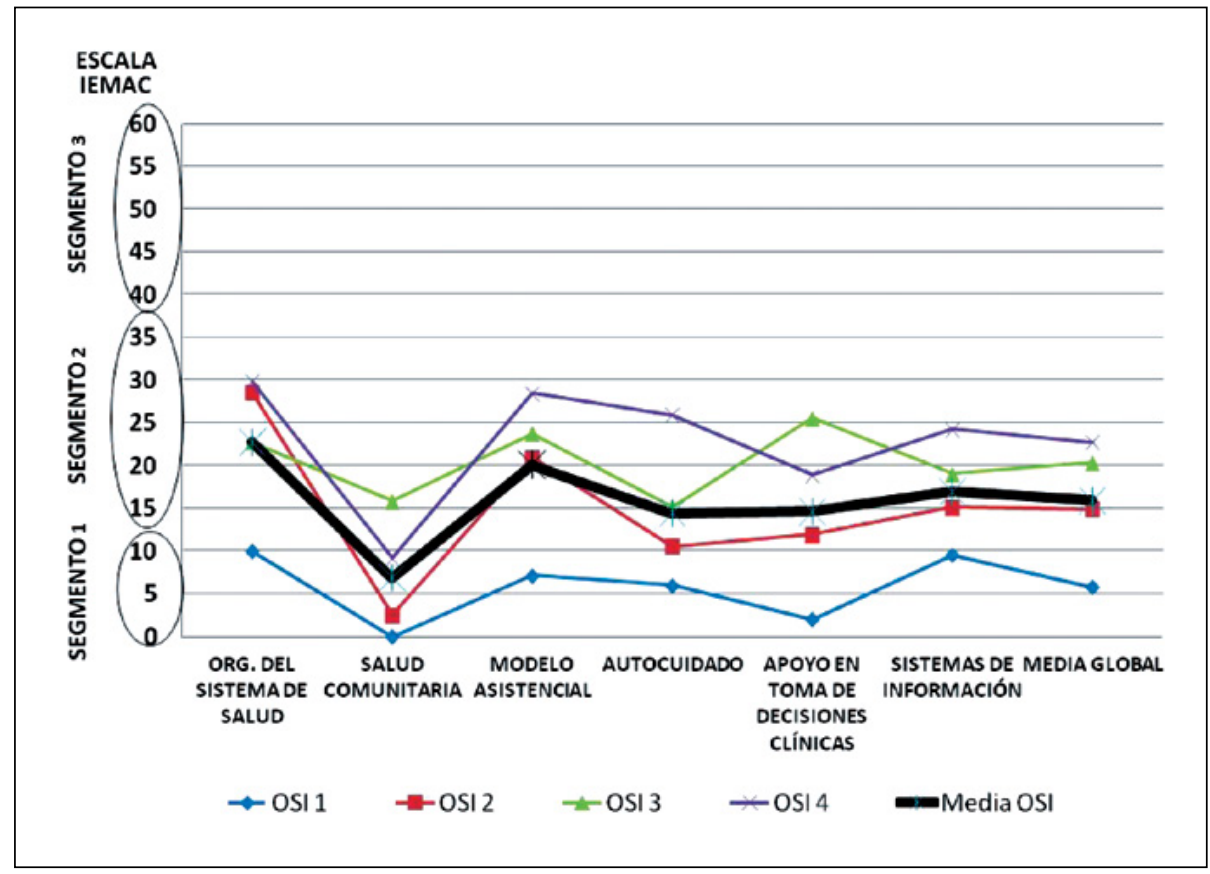

Figura 1. Puntuaciones de cada OSI por dimensión y promedio en cada dimensión.

El promedio para las cuatro OSI en IEMAC es de 16 puntos sobre 100 , lo que sitúa los enfoques de las OSI en el segundo segmento de la escala. Esto implica que las organizaciones están emprendiendo planes de acción en el ámbito de la gestión de la atención continuada, pero todavía con un despliegue limitado, de alrededor del $25 \%$ de los ámbitos relevantes y sin que se puedan aportar evidencias de haber evaluado las acciones puestas en marcha para conocer sus resultados.

En el análisis individual de cada una de las OSI (Figs. 2-3), se observó una cierta uniformidad en el perfil de desarrollo de intervenciones en las 4 OSI. La dimensión donde apareció un mayor nivel de despliegue de las intervenciones fue en relación a los componentes de la dimensión "Organización del Sistema de Salud", mientras que la peor valoración se dio en la dimensión "Salud Comunitaria". La OSI 1 no obtuvo ninguna dimensión puntuada más allá del primer segmento (0-10 puntos).
Los seis componentes que configuran la dimensión Organización del Sistema de Salud (puntuación media: 23) se sitúan en el segundo segmento de la escala, obteniendo los componentes "Compromiso de los líderes" y el "Marco estratégico" mejor puntuación. Los elementos que justificaron estas puntuaciones fueron la existencia de una Estrategia de Crónicos y la planificación estratégica conjunta entre AP y AE con objetivos comunes para los dos niveles asistenciales. El componente "políticas sociales y sanitarias" fue el menos valorado (15 puntos). Sin embargo, destacó la OSI 1 (puntuación de 25) por su enfoque en la coordinación de cuidados sociosanitarios para el paciente dependiente. La dimensión "Salud Comunitaria” (puntuación media: 7) destacó por su bajo nivel de desarrollo. Únicamente la OSI 3 había iniciado la senda de la cooperación con los recursos comunitarios, aunque de momento no se habían desplegado los planes o proyectos. En la dimensión "Modelo Asistencial" se alcanzaron de promedio 20 


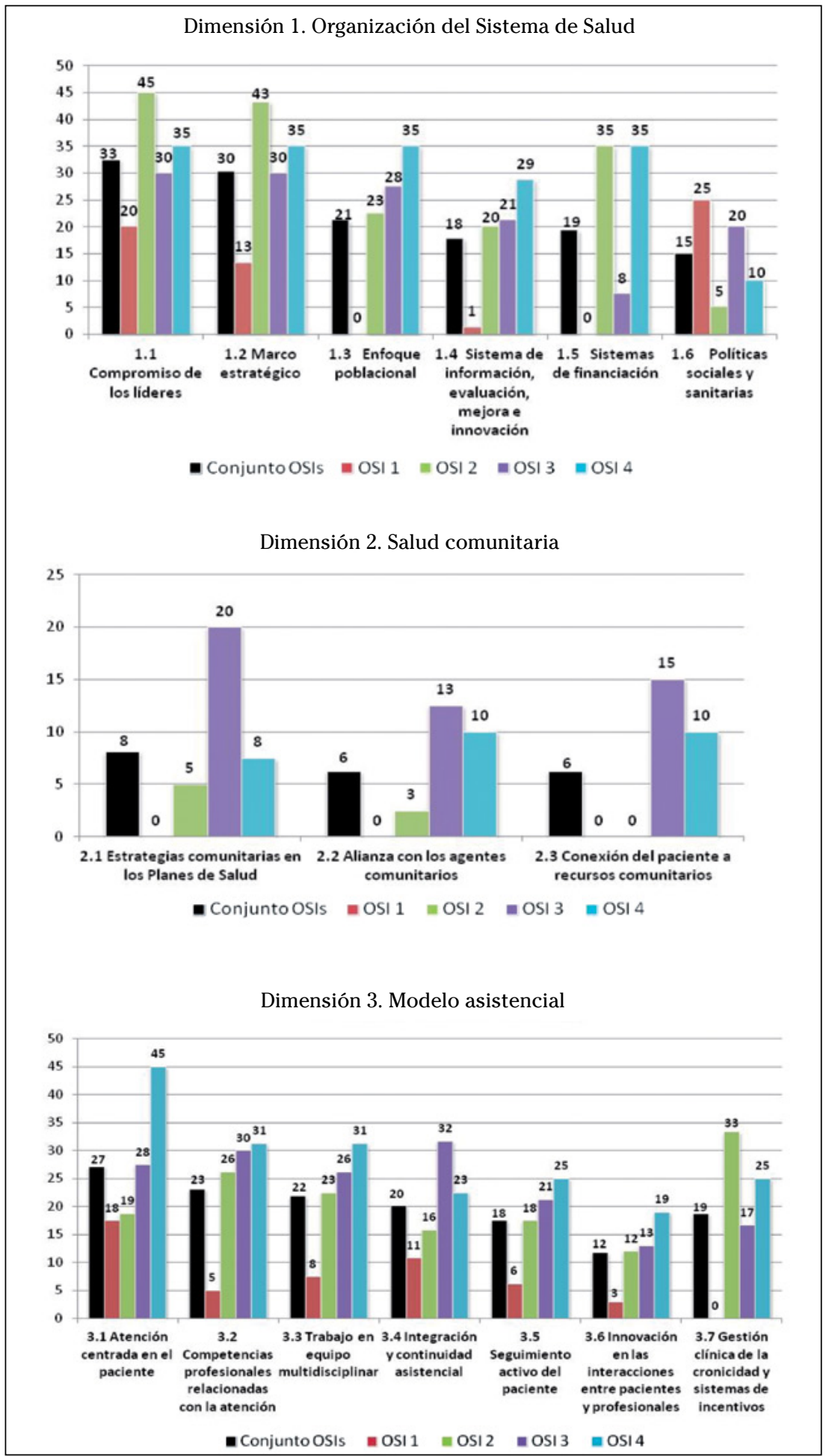

Figura 2. Puntuaciones por componente para cada OSI y para el conjunto de OSI (1) 


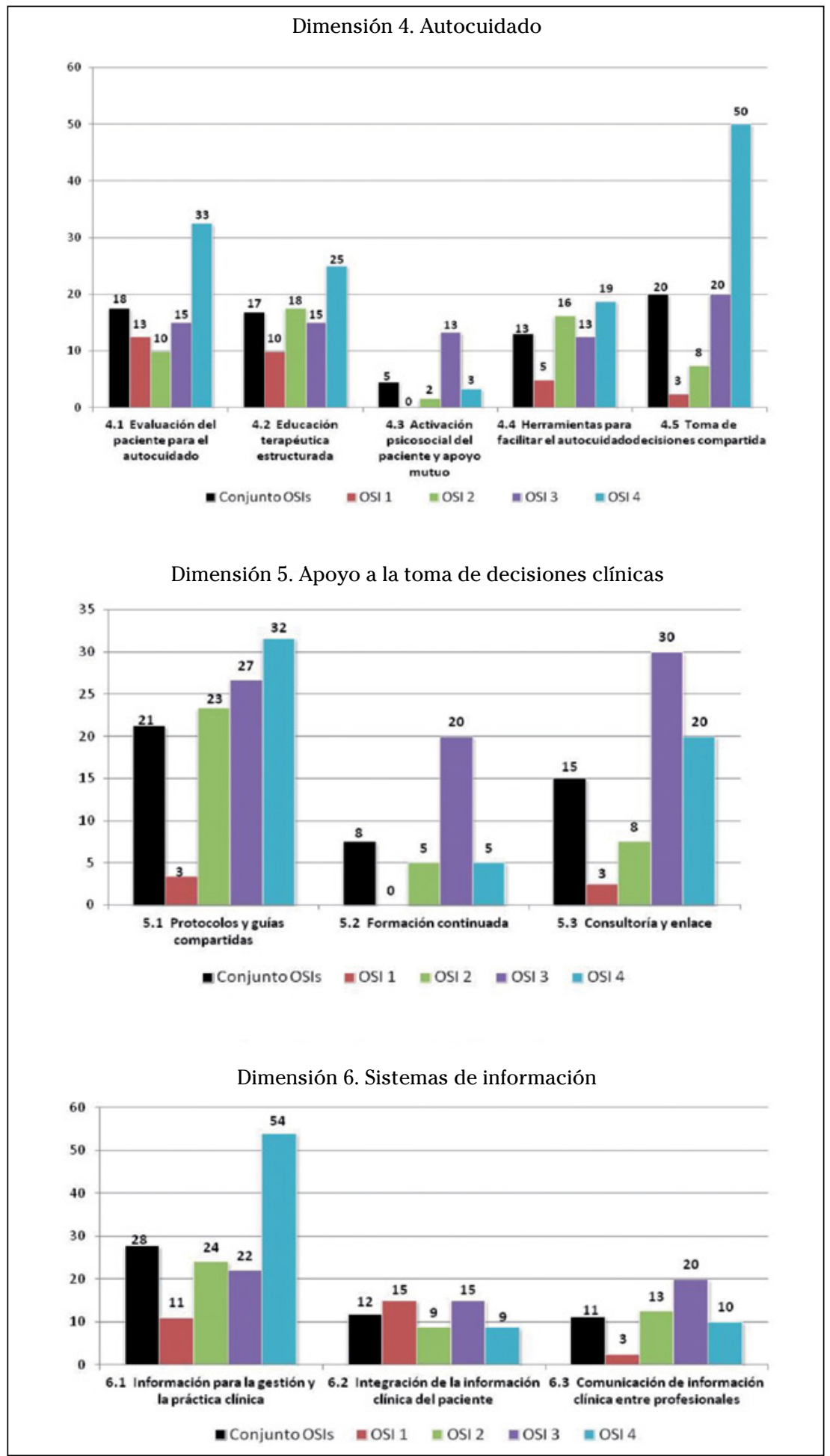

Figura 3. Puntuaciones por componente para cada OSI y para el conjunto de OSI (2) 
puntos. La existencia de proyectos que incorporan nuevas competencias profesionales en enfermería, la existencia de médicos internistas de referencia trabajando estrechamente con profesionales de AP para la atención al paciente pluripatológico ${ }^{24}$ y la elaboración de rutas asistenciales, entre otras, son las iniciativas que respaldaron las puntaciones relativamente elevadas en los componentes: "Atención centrada en el paciente", "Competencias profesionales relacionadas con la atención" e "Integración y continuidad asistencial". Las OSI han implantado el seguimiento telefónico del paciente (que se corresponde con el componente de "innovación en las interacciones") aunque se encuentran en pleno despliegue. La "Activación psicosocial del paciente" (dimensión 4: "Autocuidado" que alcanzó una puntuación promedio de 14 puntos) fue el componente menos desarrollado de los 27 de los que consta IEMAC. Destacó, en la OSI 4, como punto fuerte, los enfoques desplegados en cuanto a la "Toma de decisiones compartidas". En la dimensión 5 de "Apoyo en la toma de decisiones clínicas" (puntuación promedio de 15 puntos), el componente "Formación continuada" fue el que obtuvo un grado de desarrollo más bajo. Los "Protocolos y guías compartidas", por su parte, respaldados por la existencia de guías de práctica clínica, fueron el componente más valorado. Por último, en la dimensión 6: "Sistemas de Información" (puntuación promedio de 15 puntos) el retorno sistemático de indicadores de evaluación clínica en las organizaciones sanitarias contribuyó a puntuaciones algo más elevadas en el componente de "Información para la gestión y la práctica clínica". La existencia de una historia clínica interoperable, que permitía acceder desde cualquier nivel asistencial a la información clínica del paciente, fue el principal argumento que utilizaron las organizaciones para justificar las puntuaciones en este componente.

La tabla 2 muestra los planes, proyectos e iniciativas referidas por las OSI que alcanzaron un mayor grado de desarrollo en los diferentes componentes.

\section{DISCUSIÓN}

Las OSI creadas en Euskadi muestran en conjunto la viabilidad de los cambios que se han producido buscando una atención integrada de la cronicidad. El nivel de despliegue alcanzado en dos años tiene dos principales limitantes. Primero, que los cambios que suponen la transformación de un modelo asistencial, junto con cambios de cultura, de estrategia y con nuevos tipos de intervenciones, son siempre procesos complejos que requieren de un marco temporal adecuado para consolidar las diferentes iniciativas emprendidas ${ }^{25}$. Segundo, que dado que no se han llevado a cabo todavía por las OSI evaluaciones sistemáticas de resultados, no pudieron superar el segundo segmento de la escala de evaluación de IEMAC que supone que se haya llevado a cabo evaluación de resultados y, por tanto, las puntuaciones son inferiores a 35 puntos en todos los casos. Estos resultados, además, destacan que las intervenciones con participación de todos los niveles asistenciales junto a los recursos de carácter comunitario, pese a su demostrada efectividad ${ }^{26,27}$, son las más complejas de implantar.

Estos resultados confirman anteriores datos hallados en otros estudios ${ }^{17}$ donde se han llevado a cabo evaluaciones conforme al CCM. Primero, en lo referente a la dificultad para desplegar el conjunto de intervenciones sugeridas en el CCM, segundo, la necesidad de una clara y decidida estrategia para promover los cambios y, tercero, la necesidad de períodos de tiempo superiores al año para consolidarlos.

Este estudio responde a la necesidad de aportar experiencias sobre cómo las políticas sanitarias están contribuyendo al cambio de estrategia en la atención a la cronicidad en línea con los estudios llevados a cabo en Canadá, Estados Unidos, Inglaterra o Nueva Zelanda ${ }^{28,29}$ y cabe esperar contribuya a identificar prioridades y posibles nuevos tipos de intervenciones en los planes de atención a la cronicidad. 
Tabla 2. Planes y proyectos referidos por las OSI que muestran mayor desarrollo por componente.

\begin{tabular}{|c|c|c|}
\hline Dimensión & Componente & Planes y proyectos destacados en las OSIs \\
\hline $\begin{array}{l}\text { 1. Organización } \\
\text { del Sistema } \\
\text { de Salud }\end{array}$ & $\begin{array}{l}\text { 1.1. Compromiso de los líderes } \\
\text { 1.2. Marco estratégico } \\
\text { 1.3. Enfoque poblacional } \\
\text { 1.4. Sistemas de información, } \\
\text { evaluación, mejora e innovación } \\
\text { 1.5. Sistemas de financiación } \\
\text { 1.6. Políticas sociales y sanitarias }\end{array}$ & $\begin{array}{l}\text { - Existencia de la "Estrategia para afrontar el reto de } \\
\text { la cronicidad en Euskadi" } \\
\text { - Estrategia de crónicos, elaboración de planes } \\
\text { estratégicos conjuntos (AP y AE) que incorporan } \\
\text { o bjetivos comunes para los dos niveles } \\
\text { asistenciales (AP y AE) } \\
\text { - Implantación de sistemas de e stratificación de la } \\
\text { población de acuerdo a su riesgo } \\
\text { - Seguimiento de indicadores conjuntos para AP y AE } \\
\text { - Financiación capitativa en: AP, consultas externas } \\
\text { y urgencias (para el resto, financiación en base a } \\
\text { activida d) } \\
\text { - Coordinación de cuidados sociosanitarios para el } \\
\text { paciente dependiente entre el ámbito sanitario y } \\
\text { social }\end{array}$ \\
\hline
\end{tabular}

$\begin{array}{ll}\begin{array}{l}\text { 2. Salud } \\ \text { comunitaria }\end{array} & \begin{array}{l}\text { 2.1. Estrategias comunitarias en los } \\ \text { planes de salud }\end{array} \\ & \begin{array}{l}\text { 2.2. Alianzas con los agentes } \\ \text { comunitarios }\end{array} \\ & \begin{array}{l}\text { 2.3. Conexión del paciente a recursos } \\ \text { comunitarios }\end{array}\end{array}$

3. Modelo 3.1. Atención centrada en el paciente asistencial 3.2. Competencias profesionales relacionadas con la atención

3.3. Trabajo en equipo multidisciplinar

3.4. Integración y continuidad asistencial

3.5. Seguimiento activo del pac iente

3.6. Innovación en las interacciones entre pacientes y profesionales

3.7. Gestión clínica de la cronicidad y sistemas de incentivos

- Figura del referente asistencial en AP y AE, plataforma multicanal para la relación no presencial con el paciente (Osare an), Plan avanzado de Cuidados Paliativos

- Desarrollo de competencias profesionales para la atención integrada: nuevas figuras en enfermería (Enfermera Gestora de Competencias avanzadas [EGCA], Enfermera Gestora de Continuidad [EGC], Enfermera Gestora de Enlace Hospitalario [EGEH])

- Elaboración de rutas asistenciales entre AP y AE, creación de Unidades de Gestión Clínica transversales

- Rutas asistenciales (DM, EPOC, IC, pluripatológicos), Programas de Atención Integral al pluripatológico (con internista de referencia), Unidades de Continuidad Asistencial para pluripatológicos

- Seguimiento telefónico del paciente

- Retorno sistemático de información al profesional

4. Autocuidado 4.1. Evaluación del paciente

4.2. Educación terapéutica

4.3. Activación psicosocial

4.4. Herramientas autocuidado

4.5. Toma de decisiones compartidas
- Valoración integral del paciente pluripatológico

- Educación grupal
5.1. Protocolos y guías compartidas

5. Apoyo a la toma de decisiones clínicas

6. Sistemas de información
5.2. Formación continuada

5.3. Consultoría y enlace
- Guías de práctica clínica

- Sesiones conjuntas AP y AE
6.1. Información para la gestión y la práctica clínica

6.2. Integración de la información clínica del paciente

6.3. Comunicación de información clínica entre profesionales
- Retorno sistemático de indicadores de evaluación clínica

- Historia clínica interoperable entre AP y AE 


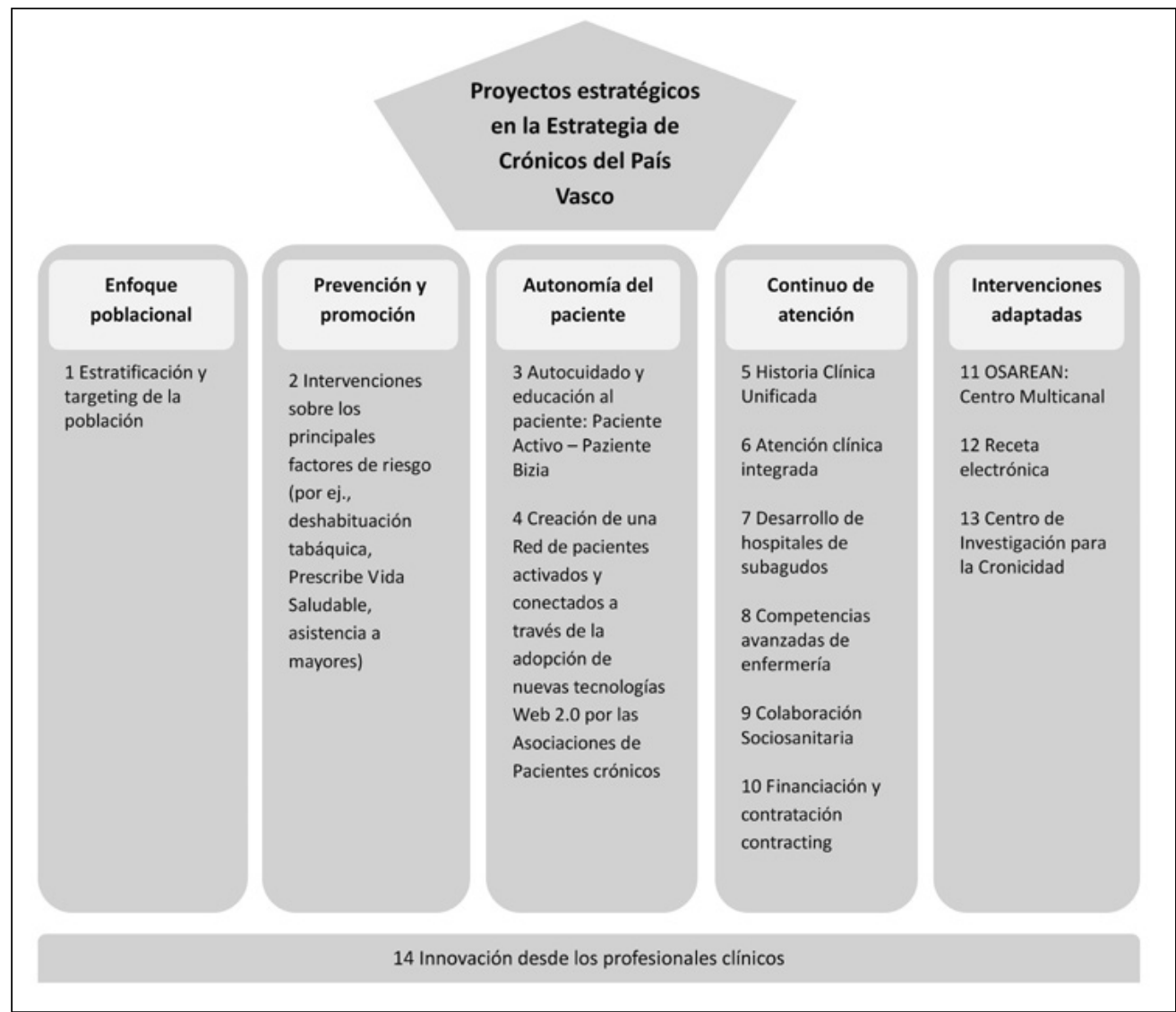

Figura 4. Políticas y proyectos estratégicos en la Estrategia de Cronicidad del País Vasco. Fuente: Estrategia para afrontar el reto de la cronicidad en Euskadi ${ }^{5}$

El cambio cultural y organizativo que supone pasar de atender la patología crónica de un paciente a atender de forma integrada e integral a un paciente pluripatológico ha sido destacado como uno de los retos más importantes de los sistemas de salud $^{30}$. Esta situación común en la práctica totalidad de los sistemas de salud es particularmente relevante para las OSI ideadas para dar una respuesta efectiva a los cambios poblacionales donde coinciden una mayor proporción de personas mayores, con un mayor número de patologías crónicas y tratamientos por paciente $\mathrm{e}^{31-34}$.

La gestión integrada a la cronicidad a partir del impulso desde 2010 de la "Estrategia para afrontar el reto de la cronicidad en Euskadi" ${ }^{5}$ puede considerarse en parte responsable de estos cambios. La estrategia se ha materializado en 14 proyectos derivados de cinco áreas de cambio o políticas de transformación: enfoque de salud poblacional estratificado, promoción y prevención de enfermedades crónicas, responsabilidad y autonomía del paciente, continuo asistencial e intervenciones eficientes adaptadas a las necesidades del paciente (Fig. 4). Este hecho justifica las mejores puntuaciones de las OSI en la dimensión 1 "Organización del Sistema de Salud". Algo parecido cabe afirmar en el caso de las dimensiones 3 "Modelo Asistencial", 5 "Apoyo en la Toma de Decisiones Clínicas" y 6 "Sistemas de Información". 
Los proyectos: "Historia clínica unificada" (que permite la interoperabilidad entre la historia clínica de AP y AE); "Atención clínica integrada" (que fomenta la integración clínica, a través del trabajo de grupos multidisciplinares entre AP y AE que definen los procesos asistenciales en torno al paciente crónico) o el de "competencias avanzadas en enfermería" (que afianza la existencia de competencias profesionales adecuadas relacionadas con la atención al crónico, fomentando la integración y la continuidad asistencial), u "Osarean" (plataforma multicanal que permite la interacción de las organizaciones sanitarias con los pacientes de manera no presencial, contribuyendo al seguimiento activo del paciente, así como a la innovación en las interacciones entre pacientes y profesionales) junto a la utilización sistematizada de guías de práctica clínica (extensión a más del $70 \%$ de los profesionales) $)^{35}$ y la protocolización de los procesos asistenciales, así como la digitalización de la historia clínica, se encuentran en la base de las valoraciones que más destacan. El efecto de la estrategia se suma a las iniciativas y buenas prácticas presentes en el sistema sanitario vasco, tales como la extensa utilización de guías de práctica clínica y la protocolización de los procesos asistenciales, así como la digitalización de la historia clínica pasa a ser interoperable entre los distintos niveles asistenciales.

La reorganización de servicios sanitarios y la introducción de cambios en la gobernanza clínica se están llevado a cabo en otros países con propósitos similares ${ }^{29}$ : integración de intervenciones desde los distintos niveles asistenciales, desarrollo de una red a nivel comunitario que logre una mayor efectividad al tiempo que se incrementa la eficiencia e instaurar un enfoque poblacional que posibilite identificar población con mayor riesgo prestando servicios acordes a dichas necesidades.

Hay que considerar algunas limitaciones en el estudio. A pesar de que se trata de organizaciones similares en tamaño y estructura y que por tanto, sus puntuaciones son comparables entre sí, las evaluaciones se han llevado a cabo en dos años distintos, 2011 y 2012, por lo que las OSI que se evaluaron antes pudieran partir con desventaja en las puntuaciones obtenidas al no haberse registrado las iniciativas emprendidas durante el último año. A medida que los proyectos orientados a la gestión de la cronicidad se desplieguen totalmente en el medio plazo, cabe esperar que se produzca una convergencia de puntuaciones en la mayoría de dimensiones. El proceso por el que se asignan las puntuaciones es un proceso autoevaluativo, según el cual el equipo directivo de cada organización puntúa en base al consenso por parte de sus miembros. Se trata, por tanto, de puntuaciones no avaladas por evaluadores externos, por lo que existe un margen evidente para la subjetividad. Ha de tenerse en cuenta que la herramienta IEMAC ofrece información sobre la situación de una organización desde la perspectiva de agentes informados que tienen una visión global de la misma, información que debería ser acompañada por otras fuentes de conocimiento de cara a poder aprehender los complejos procesos de transformación y mejora que caracterizan a las organizaciones sanitarias. Otro de los puntos que hay que considerar a la hora de interpretar las puntuaciones descritas es el carácter exigente de la escala IEMAC. Tres variables determinan los diferentes segmentos de la escala: cobertura poblacional, evaluación e integración de mejoras. La existencia de una sistemática de evaluación marca la posibilidad de acceder al tercer, cuarto y quinto segmento. Puede darse el caso de que exista una intervención con un gran despliegue poblacional (a nivel de patologías y/o profesionales implicados) pero que no está evaluada, por lo que inevitablemente su valoración queda limitada al segundo segmento.

A futuro, sería deseable profundizar en la generación de mayor evidencia sobre la eficacia y eficiencia de los modelos de gestión a crónicos, así como en la creación y refinamiento de herramientas e instrumentos que contribuyan a su implementación, facilitando de esta manera la adopción de modelos sanitarios proactivos, integrados y eficientes, que es el objetivo último de IEMAC. 


\section{BIBLIOGRAFÍA}

1. Allotey P, Reidpath DD, Yasin S, Chan CK, DeGRAFT AA. Rethinking health-care systems: a focus on chronicity. Lancet 2011; 377: 450451.

2. Jadad AR, Cabrera A, Martos F, Smith R, Lyons $\mathrm{RF}$. When people live with multiple chronic diseases: a collaborative approach to an emerging global challenge. Andalusian School of Public Health 2010.

3. TinetTI ME, FRIEd TR, Boyd CM. Designing health care for the most common chronic condition-multimorbidity. JAMA 2012; 307: 2493-2494

4. García-Goñ M, Hernández-Quevedo C, Nuño-Souinís R, Paolucci F. Pathways towards chronic care-focused healthcare systems: Evidence from Spain. Health Policy 2012; 108: 236-245.

5. Ministerio de Sanidad, Servicios Sociales e Igualdad. Estrategia para el abordaje de la cronicidad en el Sistema Nacional de Salud 2012; [consultado 20-4-2013]: Disponible enhttp://www.msssi.gob.es/organizacion/ccaa/ directorio/home.htm

6. Departamento de Sanidad y Consumo del Gobierno Vasco. Estrategia para afrontar el reto de la cronicidad en Euskadi. Gobierno Vasco 2010; [consultado 15-01-2013]: Disponible en http://es.slideshare.netlfrealday/ estrategia-para-afrontar-el-reto-de-la-cronicidad-en-euskadi

7. NuÑo-Solinís R, Orueta JF, Mateos M. An answer to chronicity in the Basque Country: primary care-based population health management. J Ambul Care Manage 2012; 35: 167-177.

8. ACUERDO de 13 de diciembre de 2010 , del Consejo de Administración del Ente Público Osakidetza-Servicio vasco de salud, por el que se crea, en el área sanitaria de Gipuzkoa, la organización sanitaria integrada Bidasoa y se suprime la organización de servicios sanitarios Hospital de Bidasoa y se modifica la organización sanitaria comarca Gipuzkoa-Este. Boletín Oficial del País Vasco 19-01-2011; 12.

9. ACUERDO de 3 de octubre de 2011, del Consejo de Administración del Ente Público Osakidetza-Servicio vasco de salud, por el que se crean, en el Área Sanitaria de Gipuzkoa, las organizaciones sanitarias integradas Alto Deba, Bajo Deba y Goierri-Alto Urola, se modifica la organización sanitaria Comarca Gipuzkoa-Este (Ekialde), que en adelante se denomina Comarca Gipuzkoa, y se suprime la organización de servicios sanitarios Comarca Gipuzkoa-Oeste (Mendebaldea). Boletín Oficial del País Vasco 16-12-11; 237.
10. VÁzquez ML, VARGas I, Nuño R, ToRo N. Organizaciones sanitarias integradas y otros ejemplos de colaboración entre proveedores. Informe SESPAS 2012. Gac Sanit 2012; 26(S): 94-101.

11. Nuño R, Coleman K, Bengoa R, Sauto R. Integrated care for chronic conditions: the contribution of the ICCC Framework. Health Policy 2012; 105: 55-64.

12. Wagner EH, Austin BT, Von KorfF M. Organizing care for patients with chronic illness. Milbank Q 1996; 74: 511-544.

13. Coleman K, Austin BT, Brach C, Wagner EH. Evidence on the Chronic Care Model in the new millennium. Health Aff 2009; 28: 75-85.

14. WHO. Innovative care for chronic conditions: building blocks for action. Global report $\mathrm{WHO} / \mathrm{NMC} / \mathrm{CCH} 2002$.

15. Barr VJ, Robinson S, Marin-Link B, Underhill L, Dotts A, Ravensdale D et al. The expanded Chronic Care Model: an integration of concepts and strategies from population health promotion and the Chronic Care Model. Hosp Q 2003; 7: 73-82.

16. Bonomi AE, Wagner EH, Glasgow RE, VonKorfF M. Assessment of chronic illness care (ACIC): a practical tool to measure quality improvement. Health Serv Res 2002; 37: 791-820.

17. Pearson ML, Wu S, Schaefer J, Bonomi AE, ShorTELL SM, MENDEL PJ et al. Assessing the implementation of the chronic care model in quality improvement collaboratives. Health Serv Res 2005; 40: 978-996.

18. Steinhaeuser J, Goetz K, Ose D, Glassen K, Natanzon I, Campbell $S$ et al. Applicability of the assessment of chronic illness care (ACIC) instrument in Germany resulting in a new questionnaire: questionnaire of chronic illness care in primary care. BMC Health Serv Res 2011; 11: 164.

19. Steurer-Stey C, Frei A, Schmid-Mohler G, MalCOLm-Kohler S, Zoller M, Rosemann T. The German version of the Assessment of Chronic Illness Care: instrument translation and cultural adaptation. J Eval Clin Pract 2012; 18: $1-4$.

20. Cramm JM, Strating MM, Tsiachisistas A, Nieboer AP. Development and validation of a short version of the Assessment of Chronic Illness Care (ACIC) in Dutch Disease Management Programs. Health Qual Life Outcomes 2011; 4: 392-349.

21. Minkman M. Towards a development model for integrated care [tesis doctoral]. Roterdam: Kluwer, Universidad Erasmus de Rotterdam 2011. 
22. Nuño-Solinís R, Fernández-Cano P, Mira JJ, ToroPolanco N, Contel JC, Gullabert Mora M et al. Desarrollo de IEMAC, un Instrumento para la Evaluación de Modelos de Atención ante la Cronicidad. Gac Sanit 2013; 27: 128-134.

23. IEMAC 1.0 [web electrónica] 2012 [consultado 15-01-2013]: Disponible en http://www. iemac.org/

24. Nuño R, SAuto R, Toro N. Integrated care initiatives in the Spanish Health System/Experiencias de integración asistencial en el Sistema Nacional de Salud de España: Abstracts from the Third Spanish Conference on Chronic Care, San Sebastián, 19-20 May 2011/ Resúmenes de Comunicaciones al III Congreso Nacional de Atención Sanitaria al Paciente Crónico, Donostia-San Sebastián, 19 y 20 de mayo del 2011. Int J Integr Care 2012; 12 (Suppl 2): e35.

25. Nuño-Solinís R, PiñERA K. Strategy for tackling the challenge of chronicity. Health Policy Monitor [revista electrónica] 2010 [consultado 15-01-2013]: Disponible en http://www. hpm.org/es16/1.pdf

26. Grau C, Fernández M. Familia y enfermedad crónica pediátrica. An Sist Sanit Navar 2010; 33: 203-212.

27. Comino E, Powell G, Krastev Y, Haas M, Christl B, FURLER J et al. A systematic review of interventions to enhance access to best practice primary health care for chronic disease management, prevention and episodic care. BMC Health Services Research 2012; 12: 415-424.

28. HAM C. The ten characteristics of the highperforming chronic care system. Health Econ Policy Law 2010; 5: 71-90.

29. Vedel I, Monette M, Beland F, Monette J, BergMAN H. Ten years of integrated care: bac- kwards and forwards. The case of the province of Québec, Canada. International Journal of Integrated Care (Special 10th Anniversary Edition) Jan-Dec 2011.

30. Tsasis P, Bains J. Chronic disease: shifting the focus of healthcare in Canada. Healthcare Quarterly 2009; 12(2): e1-11.

31. Bodenheimer T, Berry-Millett R. Follow the Money-Controlling Expenditures by Improving Care for Patients Needing Costly Services. N Engl J Med 2009; 361: 1521-1523.

32. Mira JJ, Orozco-Beltrán D, Pérez-Jover V, Martí́NEZ-Jimeno L, GiL-Guillén V, CARRATALA-Munuera $\mathrm{C}$ et al. Physician patient communication failure facilitates medication errors in older polymedicated patients with multiple comorbidities. Family Practice 2013; 30: 56-63.

33. Koper D, Kamenski G, Flamm M, Böhmdorfer B, SönNichSEN A. Frequency of medication errors in primary care patients with polypharmacy. Family Practice 2013; 30: 313-319.

34. Morilla-Herrera JC, Morales-Asencio JM, Fernández-Gallego MC, Berrobianco E, Delgado A. Utilidad y validez de un instrumento basado en indicadores de la Nursing Outcomes Classification como ayuda al diagnóstico de pacientes crónicos de Atención Primaria con gestión ineficiente de la salud propia. An Sist Sanit Navar 2011; 34: 51-61.

35. Arcelay A, Iruretagoyena ML, Reviriego E. Estudio sobre la utilización de las Guías de Práctica Clínica en Atención Especializada. Exploración de barreras y facilitadores para su implementación. Osakidetza y Osteba-Servicio de Evaluación de Tecnologías Sanitarias del País Vasco. Departamento de Salud, Gobierno Vasco (Vitoria-Gasteiz) 2013. Informe Osteba D-13-08. 
